\title{
Tanshinone IIA pretreatment attenuates ischemia/reperfusion-induced renal injury
}

\author{
YAN-MEI XU, GUO-HUA DING, JIE HUANG and YAN XIONG \\ Department of Nephrology, Renmin Hospital of Wuhan University, Wuhan, Hubei 430060, P.R. China
}

Received May 21, 2015; Accepted July 5, 2016

DOI: 10.3892/etm.2016.3674

\begin{abstract}
Tanshinone IIA is a chemical compound extracted from the root of traditional Chinese herb Salvia miltiorrhiza Bunge. Tanshinone IIA has been suggested to possess anti-inflammatory activity and antioxidizing capability. Recently, accumulating results have indicated the antitumor activity of tanshinone IIA; thus, it has attracted increasing attention. In addition, tanshinone IIA has been indicated to attenuate ischemia/reperfusion induced renal injury (I/RIRI); however, little is known regarding the underlying mechanisms involved in this process. In the present study an I/RIRI rat model was used to analyze the effects of tanshinone IIA on myeloperoxidase (MPO), TNF- $\alpha$ and IL-6 activities using ELISA kits. Furthermore, macrophage migration inhibitory factor (MIF), cleaved caspase-3, B-cell lymphoma 2 (Bcl-2) and p38 mitogen-activated protein kinase (MAPK) protein expression levels were evaluated using western blot analysis. The results indicated that tanshinone IIA protected renal function in I/RIRI rats. ELISA demonstrated that tanshinone IIA significantly reduced MIF, TNF- $\alpha$ and IL- 6 activities in I/RIRI rats. Western blot analysis showed that tanshinone IIA significantly suppressed MIF, cleaved caspase-3 and p38 MAPK protein expression levels in I/RIRI rats. The present results suggest that tanshinone IIA pretreatment attenuates I/RIRI via the downregulation of MPO expression, inflammation, MIF, cleaved caspase-3 and p38 MAPK.
\end{abstract}

\section{Introduction}

Acute renal failure (ARF) is induced by various causes, presenting as renal function rapid declining in a short time, significant impairment of glomerular filtration function, rapid increasing of blood urea nitrogen and creatinine, water, electrolyte and acid-base balance disorders, and ultimately

Correspondence to: Mr. Guo-Hua Ding, Department of Nephrology, Renmin Hospital of Wuhan University, 238 Jiefang Road, Wuhan, Hubei 430060, P.R. China

E-mail: dingdingghgh@163.com

Key words: tanshinone IIA, ischemia/reperfusion induced renal injury, myeloperoxidase, inflammation
ARF (1). Kidney injury caused by ischemia/reperfusion induced renal injury (I/RIRI) is the primary cause of ischemic ARF (2). The pathogenetic mechanism underlying I/RIRI is complicated, involving free radicals, calcium overload and energy metabolism dysfunction (3). Previous results suggest that inflammatory medium, adhesion molecules and various cytokines participate in I/RIRI (4).

I/RIRI refers to damage to tissues and organs after blood perfusion and oxygen delivery, and is the primary cause of ischemic ARF $(5,6)$. Kidneys are among the organs that I/RIRI mostly frequently occurs in (7). I/RIRI is commonly detected on ischemic diseases induced by renal blood flow transient hypoperfusion such as renal artery sclerosis, renal artery or vein embolism, severe trauma, cardiac arrest and hypovolemic shock (8). Furthermore, I/RIRI is highly frequent following organ transplantation (6). The occurrence of I/RIRI may trigger acute rejection and induce early allograft function failure, or it may lead to delayed recovery of allograft function, accelerate loss of allograft function and shorten lifespan (9). The pathogenetic mechanism underlying I/RIRI is complicated, involved in free radical, calcium overloading, energy metabolism dysfunction, inflammatory medium and adhesion molecule, upregulation of cytokines, endothelial dysfunction, abnormal blood rheology and increased apoptosis $(10,11)$.

A variety of compounds have been extracted from the roots of the herb Salvia miltiorrhiza for assessment of their clinical utility (12). In China, S. miltiorrhiza has been widely applied in the treatment of cardiovascular and cerebrovascular diseases (13). Tanshinone IIA is a key active monomer extracted from S. miltiorrhiza (14). Previous studies suggest that pre-treatment tanshinone IIA exerts a nerve protective effect against cerebral ischemia reperfusion injury $(14,15)$. The protection mechanism may involve B-cell lymphoma-2 (Bcl-2), Bcl-2-associated X protein (Bax) and TRPM7 regulation $(16,17)$. Through increasing active removal of oxygen free radicals of glutathione peroxidase, cell apoptosis may be inhibited (18). Thus, in the present study, tanshinone II A pretreatment was conducted in I/RIRI model rats, to investigate the possible underlying mechanisms, in order to provide a scientific basis for the development of a novel drug for the treatment of I/RIRI.

\section{Materials and methods}

Chemicals. The chemical structure of tanshinone IIA is indicated in Fig. 1 and purchased from Sigma-Aldrich (St. Louis, 
MO, USA). Urea Nitrogen Diacetylmonoxime Test Kit and Creatinine LiquiColor Test (Kinetic) were purchase from Tiangen Biotech. Co., Ltd. (Beijing, China). ELISA kits for the determination of myeloperoxidase (MPO), tumor necrosis factor- $\alpha$ (TNF- $\alpha$ ) and interleukin-6 (IL-6) and macrophage migration inhibition factor (MIF), and a bicinchoninic acid (BCA) protein assay kit were purchased from the Nanjing Jiancheng Bioengineering Institute (Nanjing, China).

Animals. A total of 26 adult male Sprague-Dawley rats (weight, $250 \pm 20 \mathrm{~g}$ ), were housed under a 12-h light/dark cycle at a humidity of $60-65 \%$ and temperature of $22 \pm 3^{\circ} \mathrm{C}$ with free access to food and water. The present experiment was approved by the animal experimental ethics committee of Wuhan University (Wuhan, China).

Rat model of I/RIRI. We established an I/RIRI rat model as previously described (1). Briefly, experiment rats were treated with isoflurane (2.5\%) for anesthesia. Animal body temperature was maintained during surgery. I/RIRI model was induced following a right uninephrectomy and the left kidney was ligatured using a non-traumatic aneurysm clip (FE690K; Aesculap AG, Tuttlingen, Germany) for 25 min. Reperfusion was confirmed visually when the color changed. Following surgery, experimental rats were allowed to recover and had free access to water and chow.

Groups and drug administration. Experimental rats were randomly distributed into three groups: Control group $(n=6)$, rats were intraperitoneally injected with saline; Model group ( $\mathrm{n}=10)$, I/RIRI rats were intraperitoneally injected with saline; and Tan group $(\mathrm{n}=10), \mathrm{I} / \mathrm{RIRI}$ rats were intraperitoneally injected with tanshinone IIA ( $25 \mathrm{mg} / \mathrm{kg}$ body weight) for 10 days. Following treatment, rats were sacrificed by decapitation.

Assessment of heart function. Renal tissue samples from all three groups were measured. The blood urea nitrogen (BUN) and creatinine levels were detected using commercial kits (Tiangen Biotech Co., Ltd.), according to the manufacturer's protocols.

Assessment of MPO activity. Renal tissue samples from all three groups were assessed by measuring MPO activity according to the manufacturer's instructions (Jiancheng Bioengineering Institute). MPO activity from all three groups was measured using a spectrophotometer at $460 \mathrm{~nm}$.

ELISA for assessment of TNF- $\alpha, I L-6$ and MIF activities. Renal tissue samples from all three groups were assessed by measuring TNF- $\alpha$, IL- 6 and MIF activities using a commercially obtained ELISA kits according to the manufacturer's instructions (Jiancheng Bioengineering Institute).

Western blot analysis of cleaved caspase-3, Bcl-2 and phosphorylated p38 mitogen-activated protein kinase (p-p38 MAPK). Renal injury samples from all three groups were collected and extracted using RIPA Lysis Buffer (Beyotime Institute of Biotechnology, Haimen, China) on ice for $30 \mathrm{~min}$, and analyzed using BCA protein assay kit

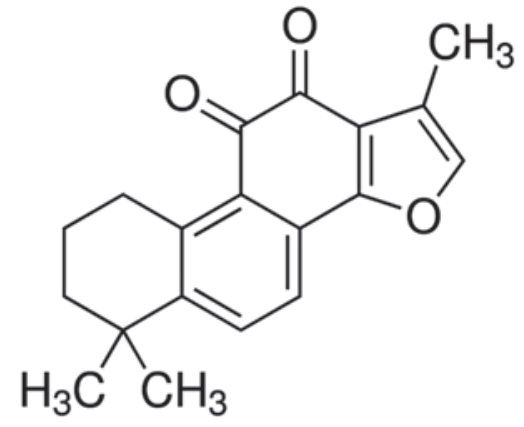

Figure 1. Chemical structure of tanshinone IIA.

(Jiancheng Bioengineering Institute). Equal quantities of total protein $(80 \mu \mathrm{g})$ from all three groups were separated using 10\% SDS-PAGE and transferred to polyvinylidene difluoride membranes (EMD Millipore, Billerica, MA, USA). The membranes were blocked in 5\% non-fat milk with phosphate-buffered saline (PBS)-Tween 20 solution. Primary antibodies against cleaved caspase- 3 (1:500; cat. no. sc-9664), Bcl-2 (1:1,000; cat. no. sc-7382), p-p38 MAPK (1:500; cat. no. sc-166182) and $\beta$-actin (1:1,000; cat. no. sc-130300) (all purchased from Santa Cruz Biotechnology, Inc., Carlsbad, CA, USA) were used at $4^{\circ} \mathrm{C}$ overnight. The membranes were incubated with $5 \%$ nonfat milk/PBS-Tween 20 containing horseradish peroxidase-conjugated secondary antibody $(1: 5,000$; goat anti-mouse IgG; cat. no. 6401-05; Amyjet Scientific Inc., Wuhan, China) for $2 \mathrm{~h}$ at $37^{\circ} \mathrm{C}$. The membranes were visualized using enhanced chemiluminescence (Thermo Fisher Scientific, Inc., Waltham, MA, USA), and assessed using a Gel Doc 2000 imaging scanner (Bio-Rad Laboratories, Inc., Hercules, CA, USA).

Statistics analysis. All data are expressed as the mean \pm standard error of the mean. Statistical analysis of data was performed using one-way analysis of variance using SPSS software, version 19.0 (SPSS, Inc., Chicago, IL, USA). A $\mathrm{P}<0.05$ was considered to indicate a statistically significant difference.

\section{Results}

Effect of tanshinone IIA on renal function. To determine the effect of tanshinone IIA on renal function of I/RIRI rats, we measured the BUN and creatinine levels with and without administration of tanshinone IIA. Fig. 2 showed I/RIRI significantly increased the BUN and creatinine levels in I/RIRI rat, compared to those of control rats. Then, relative to I/RIRI group, the BUN and creatinine levels were significantly reduced with administration of tanshinone IIA (Fig. 2).

Effect of tanshinone IIA on MPO activity. As shown in Fig. 3 , in the I/RIRI rat group, MPO activity was significantly increased compared with that of control group. The promotion of MPO activity in I/RIRI rats was significantly inhibited by the administration of tanshinone IIA, compared with the I/RIRI rat group (Fig. 3). 

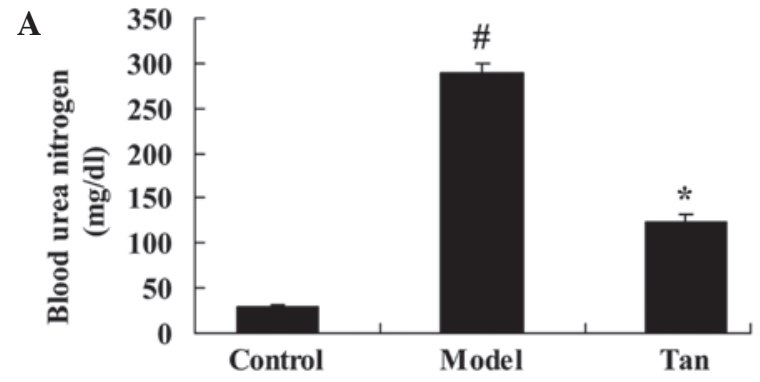

B

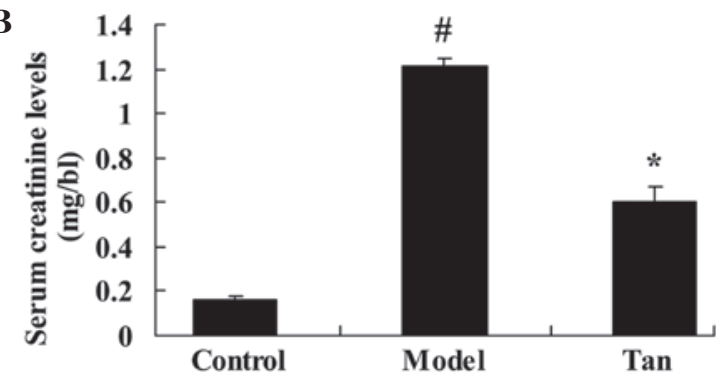

Figure 2. Effect of tanshinone IIA on (A) blood urea nitrogen and (B) creatinine levels in ischemia/reperfusion induced renal injury (I/RIRI) rat. ${ }^{\#} \mathrm{P}<0.01$ vs. Control group; " $\mathrm{P}<0.01$ vs. I/RIRI model group. Control, control group; Model, I/RIRI model group; Tan, tanshinone IIA group.

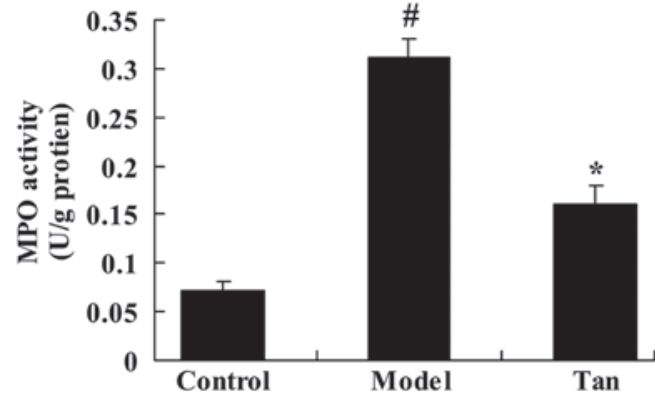

Figure 3. Effect of tanshinone IIA on MPO activity. ${ }^{\text {P }}<0.01$ vs. Control group; " $\mathrm{P}<0.01$ vs. ischemia/reperfusion induced renal injury (I/RIRI) model group. MPO, myeloperoxidase; Control, control group; Model, I/RIRI model group; Tan, tanshinone IIA group.

Effect of tanshinone IIA on proinflammatory cytokines. There was a significant increase in TNF- $\alpha$ and IL-6 activities were observed in I/RIRI rat group, compared with those of control group (Fig. 4). However, pretreatment with tanshinone IIA significantly mitigated the increase TNF- $\alpha$ and IL- 6 activities in I/RIRI rats, compared with I/RIRI rat group (Fig. 4).

Effect of tanshinone IIA on MIF activity. As shown in Fig. 5, MIF activity of I/RIRI rat was significantly higher than the control group. Relative to the I/RIRI rat group, tanshinone IIA significantly reduced MIF activity in I/RIRI rats (Fig. 5).

Effect of tanshinone IIA on cleaved caspase-3. In order to investigate the effect of tanshinone IIA on apoptosis in I/RIRI rats, cleaved caspase-3 protein expression was analyzed using western blot analysis. There was a significant increase in cleaved caspase-3 protein expression of I/RIRI rat, compared with those of control group (Fig. 6). However, the increase cleaved caspase-3 protein expression of I/RIRI rat was significantly inhibited by pretreatment with tanshinone IIA, compared with the I/RIRI rat group (Fig. 6).

Effect of tanshinone IIA on Bcl-2 protein expression. To further explore the effect of tanshinone IIA on apoptosis of I/RIRI rat, Bcl-2 protein expression was analyzed using western blot analysis. These results of western blot analysis showed that I/RIRI significantly suppressed the Bcl-2 protein expression in rat, compared with those of control group (Fig. 7). As shown in Fig. 7, the suppression of Bcl-2 protein expression was significantly increased by supplementation with tanshinone IIA, compared with the I/RIRI rat group (Fig. 7).

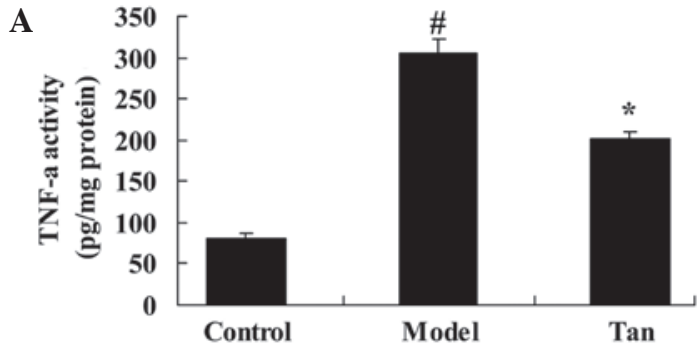

B

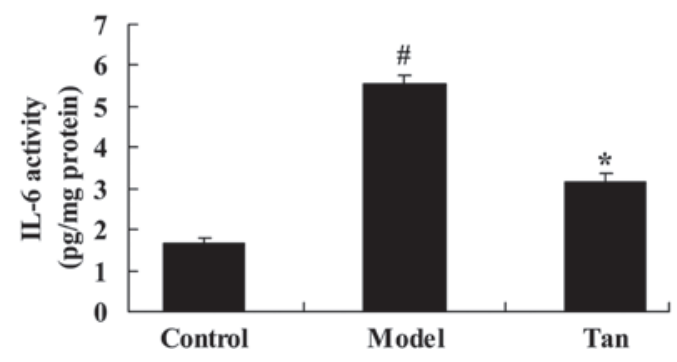

Figure 4. Effect of tanshinone IIA on (A) TNF- $\alpha$ and (B) IL-6 activities in ischemia/reperfusion induced renal injury (I/RIRI) rats. ${ }^{~} \mathrm{P}<0.01$ vs. Control group; " $\mathrm{P}<0.01$ vs. I/RIRI model group. TNF- $\alpha$, tumor necrosis factor- $\alpha$; IL-6, interleukin-6; Control, control group; Model, I/RIRI model group; Tan, tanshinone IIA group.

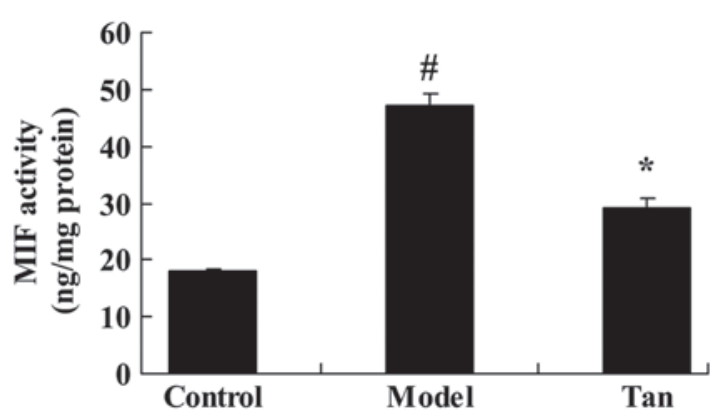

Figure 5. Effect of tanshinone IIA on MIF activity. ${ }^{\text {} P}<0.01$ vs. Control group; ${ }^{\mathrm{P}}<0.01 \mathrm{vs}$. ischemia/reperfusion induced renal injury (I/RIRI) model group. MIF, migration inhibitory factor; Control, control group; Model, I/RIRI model group; Tan, tanshinone IIA group.

Effect of tanshinone IIA on p-p38 MAPK protein expression. To further research the effect of tanshinone IIA on apoptosis of I/RIRI rat, p-p38 MAPK protein expression was analyzed using western blot analysis. Western blot analysis showed that p-p38 MAPK protein expression was significantly higher than that of the control group (Fig. 8). Following surgery, 
A Cleaved caspase-3

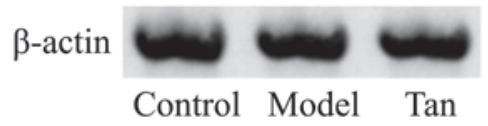

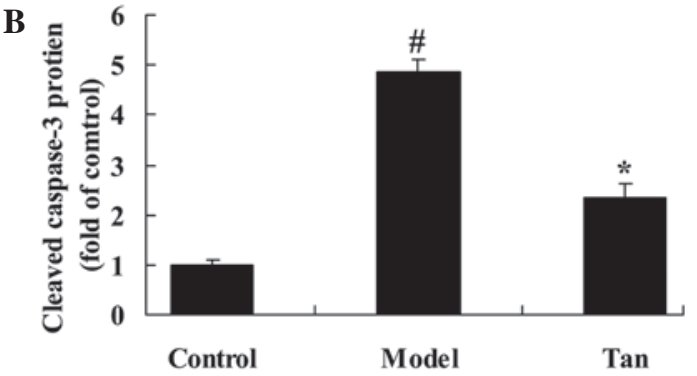

Figure 6. Effect of tanshinone IIA on cleaved caspase-3. Effect of tanshinone IIA on cleaved caspase-3 protein expression using (A) western blotting analysis, and (B) calculated cleaved caspase-3 protein expression. ${ }^{\text {P }}<0.01$ vs. Control group; ${ }^{*} \mathrm{P}<0.01$ vs. ischemia/reperfusion induced renal injury (I/RIRI) model group. Control, control group; Model, I/RIRI model group; Tan, tanshinone IIA group.

A

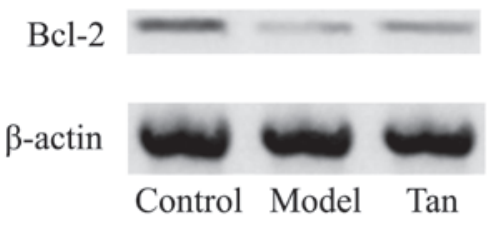

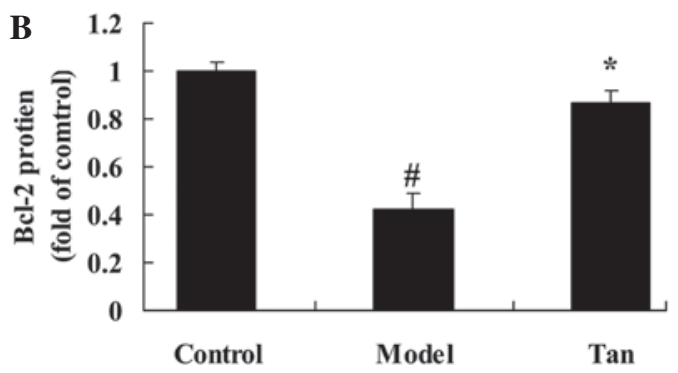

Figure 7. Effect of tanshinone IIA on Bcl-2 protein expression. Effect of tanshinone IIA on Bcl-2 protein expression using (A) western blotting analysis, and (B) calculated Bcl-2 protein expression. ${ }^{~} \mathrm{P}<0.01$ vs. Control group; ${ }^{*} \mathrm{P}<0.01$ vs. ischemia/reperfusion induced renal injury (I/RIRI) model group. Control, control group; Model, I/RIRI model group; Tan, tanshinone IIA group.

A

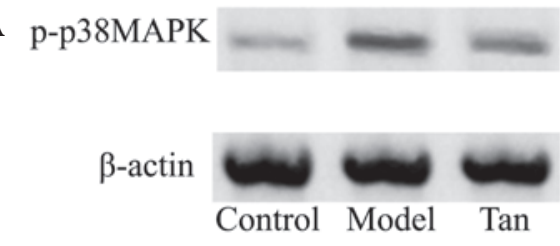

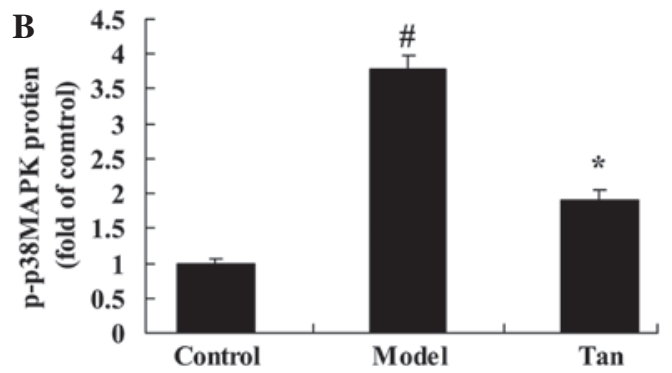

Figure 8. Effect of tanshinone IIA on p-p38 MAPK protein expression. Effect of tanshinone IIA on p-p38 MAPK protein expression using (A) western blotting analysis, and (B) calculated p-p38 MAPK protein expression. ${ }^{\#} \mathrm{P}<0.01$ vs. Control group; ${ }^{*} \mathrm{P}<0.01$ vs. ischemia/reperfusion induced renal injury (I/RIRI) model group. Control, control group; Model, I/RIRI model group; Tan, tanshinone IIA group.

tanshinone IIA significantly reduced the elevation of p-p38 MAPK protein expression in I/RIRI rat, compared with the I/RIRI rat group (Fig. 8).

\section{Discussion}

Ischemia/reperfusion is a common pathological process in clinical practice. It can be detected following acute hemorrhage, cardio-pulmonary resuscitation, cardiopulmonary bypass heart surgery and organ transplantation surgery (2). However, taking kidney transplantation as an example, ischemia/reperfusion may cause delayed recovery of transplant renal function, and in cases of inducing acute rejection may result in acute heart failure of the transplanted kidney at an early stage (5). With the application of anti-rejection drugs in clinical contexts, the occurrence rate of acute rejection has decreased to $80 \%$ (19). The year survival rate of transplant kidney has increased from 45 to $90 \%$ (19). Chronic organ damage induced by RIRI has attracted increasing attention (20). The present results indicate that tanshinone IIA pretreatment normalizes markers of renal function, and reduced BUN and creatinine levels in the I/RIRI-induced rats. These findings are consistent with previous results demonstrating that tanshinone IIA attenuates hepatic (21) and cerebral ischemia/reperfusion injury (14).

MPO primarily exists in the azurophilic granule of neutrophile granulocytes. Its activity can indicates the infiltration degree of neutrophile granulocytes in brain tissue (22). MPO activity was significantly increased in the ischemic brain tissue of rats at $24 \mathrm{~h}$ after I/RIRI, which indicates that I/RIRI is inflammatory reaction participated by neutrophile granulocyte (23). Previous studies reported that after reduction of I/RIRI, the expression level of intercellular adhesion molecule 1 (ICAM-1) or reduction of neutrophile granulocyte which can reduce I/RIRI and protective effect to renal (24). In present study, it was found that tanshinone IIA significantly reduced the I/RIRI-induced MPO activity in I/RIRI 
rats. Chen et al reported that tanshinone IIA reduced cerebral ischemia/reperfusion injury via the inhibition of MPO activity (25).

Inflammatory reaction is closely associated with I/RIRI. ICAM-1 serves an important role in the key event of inflammatory reaction and accumulation of neutrophile granulocytes (8). A large number of cytokines generated by I/RIRI, including TNF- $\alpha$, may promote the expression of ICAM-1 and induce adhesion, aggregation of neutrophile granulocytes on microvascular endothelial cells (9). It is an indicator of I/RIRI inflammatory reactions in vascular endothelial cells (10). In the present study, tanshinone IIA significantly mitigated the TNF- $\alpha$ and IL- 6 activities in I/RIRI rats. Hu et al demonstrated that tanshinone IIA protects myocardial ischemia reperfusion injury via reducing inflammatory reactions (16). Yin et al clearly show that tanshinone IIA attenuates the inflammatory response after traumatic injury of the spinal cord in adult rats (26).

The start action of immune-inflammatory responses induced by I/RIRI on multiple organ dysfunction has been gradually recognized (23). In recent years, it has been detected through investigation that MIF plays a central role in inflammatory reactions, including activation in T lymphocytes (20). MIF is generated by a variety of tissues and cells, including pituitary gland tissues, monocyte/macrophages and T lymphocytes (18). MIF is a proinflammatory mediator and a hormone derived from hypophysis (20). It can be used as a negative feedback regulator of glucocorticoid physiological activities. The present results demonstrate that tanshinone IIA significantly reduced MIF activity in I/RIRI rats. Chen et al reported that tanshinone IIA reduced cerebral ischemia/reperfusion injury via the inhibition of MIF activity (25). Furthermore, Zhang et al suggested that tanshinone IIA attenuates seawater aspiration-induced lung injury via the suppression of MIF (12).

Neuronal death following I/RIRI predominantly present as necrosis and apoptosis (6). Cell apoptosis is an active process of death under physiological and pathological conditions, and is regulated by internal and external factors (19). During I/RIRI process, necrosis of neurons and apoptosis are both observed (27). Necrocytosis is located at central area of ischemia, while cell apoptosis is mainly observed at ischemic penumbra (28). Previous studies have indicated that I/RIRI apoptosis is regulated by a variety of genes (29). Caspase- 3 is the most critical apoptotic protease in caspases cascade reaction (30). Caspase-3 plays a role in apoptosis process started by various procedures (19). It can be activated by splitting of DNA cyclin-dependent kinases to change its structure and promote cell apoptosis (29). The present data suggest that supplementation with tanshinone IIA significantly inhibited the increase cleaved caspase- 3 protein expression in I/RIRI rats. Zhang et al reported that tanshinone IIA protects against ischemia-reperfusion injury by reducing caspase-3 activity in rats (31). Zhou et al suggested that tanshinone IIA attenuates the cerebral ischemic injury via the suppression of caspases-3 (32).

Bcl-2 is an important antiapoptosis gene, particularly playing a prominent role in ischemia reperfusion injury (11). The results of this prior study showed that a large number of apoptotic cells occur at distal convoluted renal tubular after renal ischemic reperfusion. Consequently, the distal convoluted tubule after I/RIRI may play a role in anti-apoptosis via Bcl-2 overexpression and thus reduce cell damage (11). Previous studies also indicate that the antioxygenation of $\mathrm{Bcl}-2$ is indirect (11). Namely, it may inhibit the generation of superoxide anions, but not the removal of active oxygen directly (33). The superoxide anion inhibiting effect of $\mathrm{Bcl}-2$ is associated with inhibiting the release of cytochrome $\mathrm{C}$ (34). The present results suggest that supplementation of tanshinone IIA markedly increased Bcl-2 protein expression in I/RIRI rats. Zhou et al showed that tanshinone IIA protects against methylglyoxal-induced injury through Bcl-2 and p38 in human brain microvascular endothelial cells (35). Zhang et al reported that tanshinone IIA protects against Bcl-2 and Bax expression of spinal ischemia/reperfusion injury rats (18).

MAPK family is a signal conditioning enzyme connecting AFP receptor and dominant gene expression (36). It may be activated in cases of external stimulation, such as hypoxic-ischemic, inflammation, low oxygen and aglycemia. It controls the process of adaptation, proliferation, differentiation and apoptosis of cells. P38 MAPK is present within the cytoplasm, and when activated it is rapidly transferred into the nucleus and takes action on corresponding targets within cells (37). It can activate inferior kinase or a variety of transcriptional regulatory factors, such as ATF-2, Elk-1, CHOP10 and MEF-2C (36,37). Furthermore, it can activate MAPK activator protein kinase 2 and 3, and caspase family members (37). The combination of activated transcription factor and cis-regulatory elements may result in substantial genetic expression associated with apoptosis and is closely related to delayed neuronal death (36). The present results showed tanshinone IIA significantly reduced the elevation of p-p38 MAPK protein expression in I/RIRI rats. Zhou et al showed that tanshinone IIA protects against methylglyoxal-induced injury through Bcl-2 and p38 in human brain microvascular endothelial cells (35).

In conclusion, tanshinone IIA pretreatment attenuates I/RIRI, and this effect is mediated partly via he suppression of MPO, inflammatory reaction, MIF, and apoptosis-mediating caspase-3 and p-p38 MAPK in I/RIRI rats.

\section{References}

1. Hoff U, Lukitsch I, Chaykovska L, Ladwig M, Arnold C, Manthati VL, Fuller TF, Schneider W, Gollasch M, Muller DN, et al: Inhibition of 20-HETE synthesis and action protects the kidney from ischemia/reperfusion injury. Kidney Int 79: 57-65, 2011

2. Zhang J, Yao Y, Xiao F, Lan X, Yu C, Zhang Y, Jiang C, Yang J, Pei G, Li Y, et al: Administration of dexamethasone protects mice against ischemia/reperfusion induced renal injury by suppressing PI3K/AKT signaling. Int J Clin Exp Pathol 6: 2366-2375, 2013.

3. Jo SK, Ko GJ, Boo CS, Cho WY and Kim HK: Heat preconditioning attenuates renal injury in ischemic ARF in rats: Role of heat-shock protein 70 on NF-kappaB-mediated inflammation and on tubular cell injury. J Am Soc Nephrol 17: 3082-3092, 2006.

4. Tan S, Wang G, Guo Y, Gui D and Wang N: Preventive effects of a natural anti-inflammatory agent, astragaloside IV, on ischemic acute kidney injury in rats. Evid Based Complement Alternat Med 2013: 284025, 2013.

5. Wei Q, Xiao X, Fogle P and Dong Z: Changes in metabolic profiles during acute kidney injury and recovery following ischemia/reperfusion. PLoS One 9: e106647, 2014.

6. Grossini E, Molinari C, Pollesello P, Bellomo G, Valente G, Mary D, Vacca G and Caimmi P: Levosimendan protection against kidney ischemia/reperfusion injuries in anesthetized pigs. J Pharmacol Exp Ther 342: 376-388, 2012. 
7. Qin Y, Alderliesten MC, Stokman G, Pennekamp P, Bonventre JV, de Heer E, Ichimura T, de Graauw M, Price LS and van de Water B: Focal adhesion kinase signaling mediates acute renal injury induced by ischemia/reperfusion. Am J Pathol 179: 2766-2778, 2011.

8. Collino M, Benetti E, Miglio G, Castiglia S, Rosa AC, Aragno M, Thiemermann $\mathrm{C}$ and Fantozzi R: Peroxisome proliferator-activated receptor $\beta / \delta$ agonism protects the kidney against ischemia/reperfusion injury in diabetic rats. Free Radic Biol Med 50: 345-353, 2011.

9. Di Paola R, Genovese T, Impellizzeri D, Ahmad A, Cuzzocrea S and Esposito E: The renal injury and inflammation caused by ischemia-reperfusion are reduced by genetic inhibition of TNF- $\alpha$ R1: A comparison with infliximab treatment. Eur J Pharmacol 700: 134-146, 2013

10. Mizutani A, Okajima K, Uchiba M, Isobe H, Harada N, Mizutani S and Noguchi T: Antithrombin reduces ischemia/reperfusion-induced renal injury in rats by inhibiting leukocyte activation through promotion of prostacyclin production. Blood 101: 3029-3036, 2003.

11. Isaka Y, Suzuki C, Abe T, Okumi M, Ichimaru N, Imamura R, Kakuta Y, Matsui I, Takabatake Y, Rakugi H, et al: Bcl-2 protects tubular epithelial cells from ischemia/reperfusion injury by dual mechanisms. Transplant Proc 41: 52-54, 2009.

12. Zhang Y, Zhang B, Xu DQ, Li WP, Xu M, Li JH, Xie XY, Fan QX, Liu W, Mu DG, et al: Tanshinone IIA attenuates seawater aspiration-induced lung injury by inhibiting macrophage migration inhibitory factor. Biol Pharm Bull 34: 1052-1057, 2011.

13. van Poppel PC, Breedveld P, Abbink EJ, Roelofs H, van Heerde W, Smits P, Lin W, Tan AH, Russel FG, Donders R, et al: Salvia miltiorrhiza root water-extract (Danshen) has no beneficial effect on cardiovascular risk factors. A randomized double-blind cross-over trial. PLoS One 10: e0128695, 2015.

14. Chen Y, Wu X, Yu S, Fauzee NJ, Wu J, Li L, Zhao J and Zhao Y: Neuroprotective capabilities of tanshinone IIA against cerebral ischemia/reperfusion injury via anti-apoptotic pathway in rats. Biol Pharm Bull 35: 164-170, 2012.

15. Wen PY, Li J, Lu BL, Liu J, Yang FZ, Zhou L, Luo H, Li WW and Zhou J: Tanshinone IIA increases levels of NeuN, protein disulfide isomerase, and $\mathrm{Na}^{+} / \mathrm{K}^{+}$-ATPase and decreases evidence of microglial activation after cerebral ischemic injury. Neuroreport 27: 435-444, 2016.

16. Hu H, Zhai C, Qian G, Gu A, Liu J, Ying F, Xu W, Jin D, Wang H, $\mathrm{Hu} \mathrm{H}$, et al: Protective effects of tanshinone IIA on myocardial ischemia reperfusion injury by reducing oxidative stress, HMGB1 expression and inflammatory reaction. Pharm Biol 53: $1752-1758,2015$

17. Zhang L, Gan W and An G: Influence of tanshinone IIA on heat shock protein $70, \mathrm{Bcl}-2$ and Bax expression in rats with spinal ischemia/reperfusion injury. Neural Regen Res 7: 2882-2888, 2012.

18. Wang S, Zang W, Yang Y, Zhang Q, Zhao M, Gao Z, Li G, Meng Q, Liu Q and Zheng X: Tanshinone IIA and Baicalin inhibiting the formation of benzo[a]pyrene and benzo[a]pyrene induced cytotoxicity: Correlation with scavenging free radical. Environ Toxicol Pharmacol 36: 403-410, 2013.

19. El Morsy EM, Ahmed MA and Ahmed AA: Attenuation of renal ischemia/reperfusion injury by açaí extract preconditioning in a rat model. Life Sci 123: 35-42, 2015.

20. Wang F, Yu G, Liu SY, Li JB, Wang JF, Bo LL, Qian LR, Sun XJ and Deng XM: Hydrogen-rich saline protects against renal ischemia/reperfusion injury in rats. J Surg Res 167: e339-e344, 2011.

21. Qi YY, Xiao L, Zhang LD, Song SH, Mei Y, Chen T, Tang JM, Liu F, Ding GS, Shi YZ and Wang QX: Tanshinone IIA pretreatment attenuates hepatic ischemia-reperfusion. Front Biosci (Elite Ed) 4: 1303-1313, 2012.
22. Wang H, Xu L, Zhao J, Wang D, Guo R, Wang J, Gong W, Liu T, Zhang Y and Dong L: Regulatory mechanism of pyrrolidine dithiocarbamate is mediated by nuclear factor- $\kappa \mathrm{B}$ and inhibits neutrophil accumulation in ARDS mice. Exp Ther Med 8: 614-622, 2014.

23. Kurcer Z, Oguz E, Ozbilge H, Baba F, Aksoy N, Celik H, Cakir H and Gezen MR: Melatonin protects from ischemia/reperfusion-induced renal injury in rats: This effect is not mediated by proinflammatory cytokines. J Pineal Res 43: 172-178, 2007.

24. Ragab D, Abdallah DM and El-Abhar HS: Cilostazol renoprotective effect: Modulation of PPAR- $\gamma$, NGAL, KIM-1 and IL-18 underlies its novel effect in a model of ischemia-reperfusion. PLoS One 9: e95313, 2014.

25. Chen Y, Wu X, Yu S, Lin X, Wu J, Li L, Zhao J and Zhao Y: Neuroprotection of tanshinone IIA against cerebral ischemia/reperfusion injury through inhibition of macrophage migration inhibitory factor in rats. PLoS One 7: e40165, 2012.

26. Yin X, Yin Y, Cao FL, Chen YF, Peng Y, Hou WG, Sun SK and Luo ZJ: Tanshinone IIA attenuates the inflammatory response and apoptosis after traumatic injury of the spinal cord in adult rats. PLoS One 7: e38381, 2012.

27. Yang CC, Lin LC, Wu MS, Chien CT and Lai MK: Repetitive hypoxic preconditioning attenuates renal ischemia/reperfusion induced oxidative injury via upregulating HIF-1 alpha-dependent bcl-2 signaling. Transplantation 88: 1251-1260, 2009.

28. Yan XG, Cheng BH, Wang X, Ding LC, Liu HQ, Chen J and Bai B: Lateral intracerebroventricular injection of Apelin-13 inhibits apoptosis after cerebral ischemia/reperfusion injury. Neural Regen Res 10: 766-771, 2015.

29. Ben Mkaddem S, Pedruzzi E, Werts C, Coant N, Bens M, Cluzeaud F, Goujon JM, Ogier-Denis E and Vandewalle A: Heat shock protein gp96 and NAD(P)H oxidase 4 play key roles in Toll-like receptor 4-activated apoptosis during renal ischemia/reperfusion injury. Cell Death Differ 17: 1474-1485, 2010.

30. Zhao WY, Han S, Zhang L, Zhu YH, Wang LM and Zeng L: Mitochondria-targeted antioxidant peptide SS31 prevents hypoxia/reoxygenation-induced apoptosis by down-regulating p66Shc in renal tubular epithelial cells. Cell Physiol Biochem 32: 591-600, 2013.

31. Zhang MQ, Zheng YL, Chen H, Tu JF, Shen Y, Guo JP, Yang XH, Yuan SR, Chen LZ, Chai JJ, et al: Sodium tanshinone IIA sulfonate protects rat myocardium against ischemia-reperfusion injury via activation of $\mathrm{PI} 3 \mathrm{~K} / \mathrm{Akt} / \mathrm{FOXO} \mathrm{A} / \mathrm{Bim}$ pathway. Acta Pharmacol Sin 34: 1386-1396, 2013.

32. Zhou L, Bondy SC, Jian L, Wen P, Yang F, Luo H, Li W and Zhou J. Tanshinone IIA attenuates the cerebral ischemic injury-induced increase in levels of GFAP and of caspases-3 and -8. Neuroscience 288: 105-111, 2015.

33. Chien CT, Shyue SK and Lai MK: Bcl-xL augmentation potentially reduces ischemia/reperfusion induced proximal and distal tubular apoptosis and autophagy. Transplantation 84: 1183-1190, 2007.

34. Chien CT, Chang TC, Tsai CY, Shyue SK and Lai MK: Adenovirus-mediated bcl-2 gene transfer inhibits renal ischemia/reperfusion induced tubular oxidative stress and apoptosis. Am J Transplant 5: 1194-1203, 2005.

35. Zhou WJ, Gui QF, Wu Y and Yang YM: Tanshinone IIA protects against methylglyoxal-induced injury in human brain microvascular endothelial cells. Int J Clin Exp Med 8: 1985-1992, 2015.

36. Lempiäinen J, Finckenberg P, Mervaala EE, Storvik M, Kaivola J, Lindstedt K, Levijoki J and Mervaala EM: Dexmedetomidine preconditioning ameliorates kidney ischemia-reperfusion injury. Pharmacol Res Perspect 2: e00045, 2014.

37. Park KM, Kramers C, Vayssier-Taussat M, Chen A and Bonventre JV: Prevention of kidney ischemia/reperfusion-induced functional injury, MAPK and MAPK kinase activation and inflammation by remote transient ureteral obstruction. J Biol Chem 277: 2040-2049, 2002. 